\title{
Anticipating the Novel Coronavirus Disease (COVID-19) Pandemic
}

\author{
Taranjot Kaur ${ }^{1}$, Sukanta Sarkar ${ }^{1}$, Sourangsu Chowdhury ${ }^{2}$, Sudipta Kumar Sinha ${ }^{3}$, \\ Mohit Kumar Jolly ${ }^{4}$ and Partha Sharathi Dutta ${ }^{1 *}$
}

${ }^{1}$ Department of Mathematics, Indian Institute of Technology Ropar, Rupnagar, India, ${ }^{2}$ Department of Atmospheric Chemistry, Max Planck Institute for Chemistry, Mainz, Germany, ${ }^{3}$ Department of Chemistry, Indian Institute of Technology Ropar, Rupnagar, India, ${ }^{4}$ Centre for BioSystems Science \& Engineering, Indian Institute of Science, Bengaluru, India

\section{OPEN ACCESS}

Edited by:

Zisis Kozlakidis,

International Agency for Research on

Cancer (IARC), France

Reviewed by:

Sérvio Pontes Ribeiro,

Universidade Federal de Ouro Preto,

Brazil

Enahoro Iboi,

Spelman College, United States

*Correspondence:

Partha Sharathi Dutta parthasharathi@iitrpr.ac.in

Specialty section:

This article was submitted to Infectious Diseases - Surveillance,

Prevention and Treatment,

a section of the journal

Frontiers in Public Health

Received: 04 June 2020

Accepted: 12 August 2020

Published: 03 September 2020

Citation:

Kaur T, Sarkar S, Chowdhury S, Sinha SK, Jolly MK and Dutta PS

(2020) Anticipating the Novel

Coronavirus Disease (COVID-19)

Pandemic.

Front. Public Health 8:569669. doi: 10.3389/fpubh.2020.569669
The COVID-19 outbreak was first declared an international public health, and it was later deemed a pandemic. In most countries, the COVID-19 incidence curve rises sharply over a short period of time, suggesting a transition from a disease-free (or low-burden disease) equilibrium state to a sustained infected (or high-burden disease) state. Such a transition is often known to exhibit characteristics of "critical slowing down." Critical slowing down can be, in general, successfully detected using many statistical measures, such as variance, lag-1 autocorrelation, density ratio, and skewness. Here, we report an empirical test of this phenomena on the COVID-19 datasets of nine countries, including India, China, and the United States. For most of the datasets, increases in variance and autocorrelation predict the onset of a critical transition. Our analysis suggests two key features in predicting the COVID-19 incidence curve for a specific country: (a) the timing of strict social distancing and/or lockdown interventions implemented and (b) the fraction of a nation's population being affected by COVID-19 at that time. Furthermore, using satellite data of nitrogen dioxide as an indicator of lockdown efficacy, we found that countries where lockdown was implemented early and firmly have been successful in reducing COVID-19 spread. These results are essential for designing effective strategies to control the spread/resurgence of infectious pandemics.

Keywords: COVID-19, critical transitions, indicators of critical slowing down, social distancing policies, nonpharmaceutical interventions

\section{INTRODUCTION}

The outbreak of the COVID-19 disease caused by a novel pathogenic coronavirus (SARS-CoV-2), which began in Wuhan, China, in December 2019, is a global challenge for the healthcare, economy and the society (1). The World Health Organization (WHO) assessed the epidemics of the disease (COVID-19) and declared it a Public Health Emergency of International Concern (PHEIC) (2). Since the Wuhan outbreak, nearly all the United Nations member countries have experienced a rapid spread of the virus and have been taking preventive measures to overcome the threats posed by the pandemic (3). Over the past years, several waves of viruses, such as influenza, cholera, and HIV have transmitted across the world to pose a significant threat to human health. Investigations on the interventions of these outbreaks have increased within the predictive theory of infectious diseases. Importantly, prior understanding of the epidemic spread of COVID-19 can provide an effective mitigation policy. 
The COVID-19 disease can spread in a population through infected symptomatic/asymptomatic individuals who come into contact directly or indirectly (4). Concerned with the public health and well-being affected due to COVID-19, various countries have thus adopted comprehensive clinical and non-pharmaceutical strategies. The non-pharmaceutical interventions have included social distancing, such as the closure of schools, banning of large gatherings, isolation of symptomatic individuals, and monitoring of travelers, particularly those from COVID-19 hotspots (5-8). There also exists evidence of similar non-pharmaceutical interventions used to mitigate the 1918 influenza pandemic $(9,10)$. Evidence also highlights the importance of mitigation interventions in controlling the transmission of the SARS-CoV-2 virus $(6,11,12)$. Nonetheless, the timing of the implementation of strategies varies between countries and can significantly influence the incidence curve of the epidemic (13).

The COVID-19 incidence curve of total confirmed cases for many countries initially demonstrates a gradual increase near the start of the epidemic and is often followed by a sudden shoot or a transition to a supercritical state (14-18), as the disease spreads (major outbreak due to human-to-human transmission). This sudden transition places a considerable burden on the limited availability of the public health resources required to treat the disease and inhibit its further spread. Most of the studies on sudden transitions concern catastrophic shifts associated with a saddle-node bifurcation; however, epidemic transitions are non-catastrophic and associated with a transcritical bifurcation $(17,19)$. In general, an epidemic transition occurs when the basic reproduction number (or $\mathcal{R}_{0}$ ) of the disease becomes $>1$ and a population moves from a subcritical to a supercritical state. In many countries, however, major outbreaks of COVID-19 did not initially occur, though the $\mathcal{R}_{0}$ of the disease is known to be more than one from the very beginning (20). In fact, this may be associated with a tipping delay where a population faces the first major outbreak at a higher value of $\mathcal{R}_{0}$ than one $(14,21)$, impending our ability to mitigate. It is thus crucial to anticipate this precarious transition to take effective controlling measures for the outbreak. There exists a rich history of investigations that can predict processes that could lead to ecological outbreaks $(19,22-24)$. Theory suggests applicability of a variety of leading generic indicators, widely known as Early Warning Signals (EWSs) (e.g., variance, autocorrelation, skewness, and kurtosis), to identify the proximity of a system to such a critical transition $(22,23,25,26)$. For instance, in time series data following ancient abrupt climate shifts, EWSs could be identified before the critical transition took place (27). Similarly, EWSs were seen in the resurgence of malaria in Kericho, Kenya (18).

EWSs are hallmarks of critical slowing down (CSD) of a system as it approaches a catastrophic/non-catastrophic transition. The phenomenon of CSD is caused by to the loss of resilience in the system such that even small disturbances can invoke an often irreversible transition to an alternative stable state (28-31). In particular, dynamical systems are continuously subject to shocks that may be extrinsic or intrinsic perturbations. In epidemiological theory, intrinsic perturbations can be determined by the pathogen's novelty in a new host, which may depend upon various health factors associated with the host. Furthermore, the mode of transmission, person-to-person contact, and number of imported cases may account for external perturbations for disease spread. Increased perturbations may drive a system far from its original state and can increase the time required for fluctuations in the number of cases to dampen. The system thus loses its resilience, as it may eventually diverge at a transition from a low burdened to a high burdened state. The phenomenon of CSD can be captured as a large time taken by a system to return to its previous states due to which the rate of return of a system decreases prior to a transition. Moreover, it leads to an increase in the short-term memory of a system, this feature can be identified by the changes in the correlation structure of a time series preceding a critical transition $(22,23$, $26,32,33)$.

Model based epidemiological investigations predict the phenomenon of CSD preceded by the epidemic transitions (14, $15,34)$. These studies are built on the applicability of CSD-based EWSs to anticipate disease emergence. However, construction of emerging disease models can be complicated partly due to non-linearity in many natural systems. Additionally, data availability of key epidemiological parameters, such as rate and mode of transmission, duration of infection, and the novelty of the pathogen in a new host, can pose a barrier toward disease predictive theory. The key support of CSD-based EWSs analyzes over modeling prediction is that it does not require comprehensive data calibration and can be calculated using observed data. Furthermore, it is studied that imperfections in the disease data does not form a barrier in applicability of EWSs (15).

To mitigate the epidemic, China strictly restricted public movement and followed with measures of quarantine and symptomatic isolation 24 days after (i.e., January 23) the arrival of the first reported case. The total reported cases (confirmed) at the time of the lockdown were nearly 623 (accounting for $\sim 4.4732 \times 10^{-7}$ of the total population). The daily increase in the number of confirmed cases in China was saturated in mid-March, hence flattening the incidence curve of the total confirmed cases. European countries adopted different non-pharmaceutical measures to intervene in the disease transmission. The spread began later in Italy compared to China; however, the strict interventions were initiated on March 9, which marks a gap of nearly 40 days from the first reported case with $\approx 1.22 \times 10^{-4}$ proportion of the cases. Spain, which is continued to suffer severely by the virus, reported its first infected case on February 1 and took nearly 45 days when the proportion of affected cases was more than $9.05 \times 10^{-5}$, to put the country into lockdown (see Table S1 in Supplementary Material). India confirmed its first case on January 30 and prompted a "Janata curfew" on March 22 followed by a nationwide lockdown on March 25 for complete cessation of public contacts (nearly 55 days after the first case being reported). The proportion of cases were $\sim 2.36 \times$ $10^{-7}$ of its population (COVID-19-infected cases), while this proportion was more than $1.7 \times 10^{-4}$ in the US. Therefore, it is essential to understand how prolonged gaps between the arrival of the epidemic and non-pharmaceutical interventions, such as quarantining/social distancing can influence public health and 
the environment at a national as well as a global scale. Of greater interest is outlining whether the EWSs can be useful to stifle the spread of an epidemic.

In this work, we analyze how the timing of strict controlling strategies influence the COVID-19 incidence curve of the total confirmed cases in different countries. We first use the "change in the gradient" analysis (for details see Section 4: Detection of the Transition Phase in Supplementary Material), to estimate the emergence of the transition phase in incidence curves. The occurrence of CSD is then analyzed using the data prior to the transition. We calculate the variance and lag-1 autocorrelation function of the time series data of the cumulative confirmed cases each in nine different countries. Our work suggests that the dynamics of incidence curve in the initial days (depending upon the country), since the first reported case, can signal an upcoming sudden rise in the cumulative number of infected cases. Preliminary intervention is thus crucial for an effective and timely containment of the disease emergence or resurgence. Delay in the strict surveillance and control measures can increase the time to contain the spread, which in turn will affect a larger proportion of the population. Furthermore, the proportion of the affected cases on the commencement of public health measures plays a significant role in containing the epidemic in each country. The time gap of implementation of interventions from the arrival of the first case is almost similar for many countries, such as Italy, India and Germany. However, the EWSs depict an upcoming rise in Italy and Germany relatively earlier than in India. The relatively low proportion of the affected cases in the case of India compared to Italy or Germany can be a significant factor, explaining a slow rise for India but a relatively disruptive situation in the other countries. A combination of these two factors for India may thus restrict the extent of COVID-19 spread in the country, as compared to many other countries across the world. Importantly, despite keeping control of the situation up to April 29, India, having greater carrying capacity for the disease and several challenges to sanitization control (35), needs strict and highly effective interventions for continued suppression in the daily number of cases. We conclude that model-independent forecasting systems can be applied to clinical datasets for predictability of the disease re-occurrence and formulate control policies.

\section{RESULTS}

We obtain the datasets of the cumulative number of the COVID19 cases from the date of reporting of the first affected person up to April 29, 2020, for India, China, South Korea, the United States (US), Singapore, Germany, Italy, the United Kingdom (UK), and Spain (for the data source see Materials and Methods). Figure 1 depicts the incidence curve of the affected population in each of these countries. Interestingly, it is noted that the incidence curve of the confirmed cases follows a slow increment during initial time period ranging from $\approx 20$ to 50 days for different countries, which can be interpreted as a time window to control the epidemic promptly and effectively. Since human-to-human contact is a leading transmitter of the disease, by-passing a certain threshold of infected cases, the incidence curve thus shows an increasing slope and finally depicts a transition in the number of infected cases (see Figure 1) (36). It is important to note that the growth in number of cases for China and South Korea, countries that initiated public monitoring/social distancing actions relatively earlier than the other countries, saturates after nearly 3-4 weeks from the initiation of the lockdown. The shift of the COVID-19 from a low-burden to a high-burden state can be associated with the phenomenon of critical transition. We thus employ statistical methods that can monitor the onset of the transition phase and provide insights into the incidence curve so as to suggest establishing worldwide disease elimination campaigns.

\subsection{Signals of Critical Slowing Down}

To estimate statistical indicators anticipating the upcoming shifts in each country, we consider the data of the cumulative daily number of COVID-19 cases before a transition is detected in the incidence curve of the epidemic (shaded regions in Figure 1) (for most of the countries, a transition threshold is detected by a gradient change analysis, for details see Section 4: Detection of the Transition Phase and Figure S5 in Supplementary Material). To examine whether the system slows down to recover from perturbation while approaching the transition, we calculate the variance and autocorrelation at first lag $[\mathrm{ACF}(1)]$ of each extracted data for all the nine countries (see Materials and Methods). We have also calculated a few other generic EWSs of CSD, like density ratio, skewness, and kurtosis (for details see Section 1: Early Warning Indicators and Figure S1 in Supplementary Material). CSD is reflected in systems near a critical transition through an increase in the variance and autocorrelation. We observe that the short-term memory of the time series data exhibits an increasing trend in most of the countries (Figure 2). However, there are no positive signals of CSD exhibited by $\mathrm{ACF}(1)$ for the datasets of India or Italy (Figures 2J,P). The increase in the variance forewarns a sudden rise in the number of the COVID-19 cases for these countries. Furthermore, the strength of the signals varies among countries depending upon the datasets determining the cumulative number of affected populations in individual countries. For instance, we observe a weak increase in variance in case of Singapore, and the trends in China and the US are observed to be very strong, with $\mathrm{ACF}(1)$ approaching close to 1 (see Figures $\mathbf{2 K}, \mathbf{M}$ ) (32). Since the time lag of up to almost 2 weeks is expected for the detection of symptomatic cases (37), the analyses suggest that the total cases gathered when the phenomenon of CSD is observed must have been infected with the disease around 2 weeks ago. Thus, early preventive and surveillance strategies can be capable of suppressing the severity of COVID-19 outbreak (38).

\subsection{EWSs and Enforcement of Interventions}

The timing of intervention measures varies among the countries. Notice that, apart from China and South Korea, in other countries, EWS analyses are carried out using the data before the 

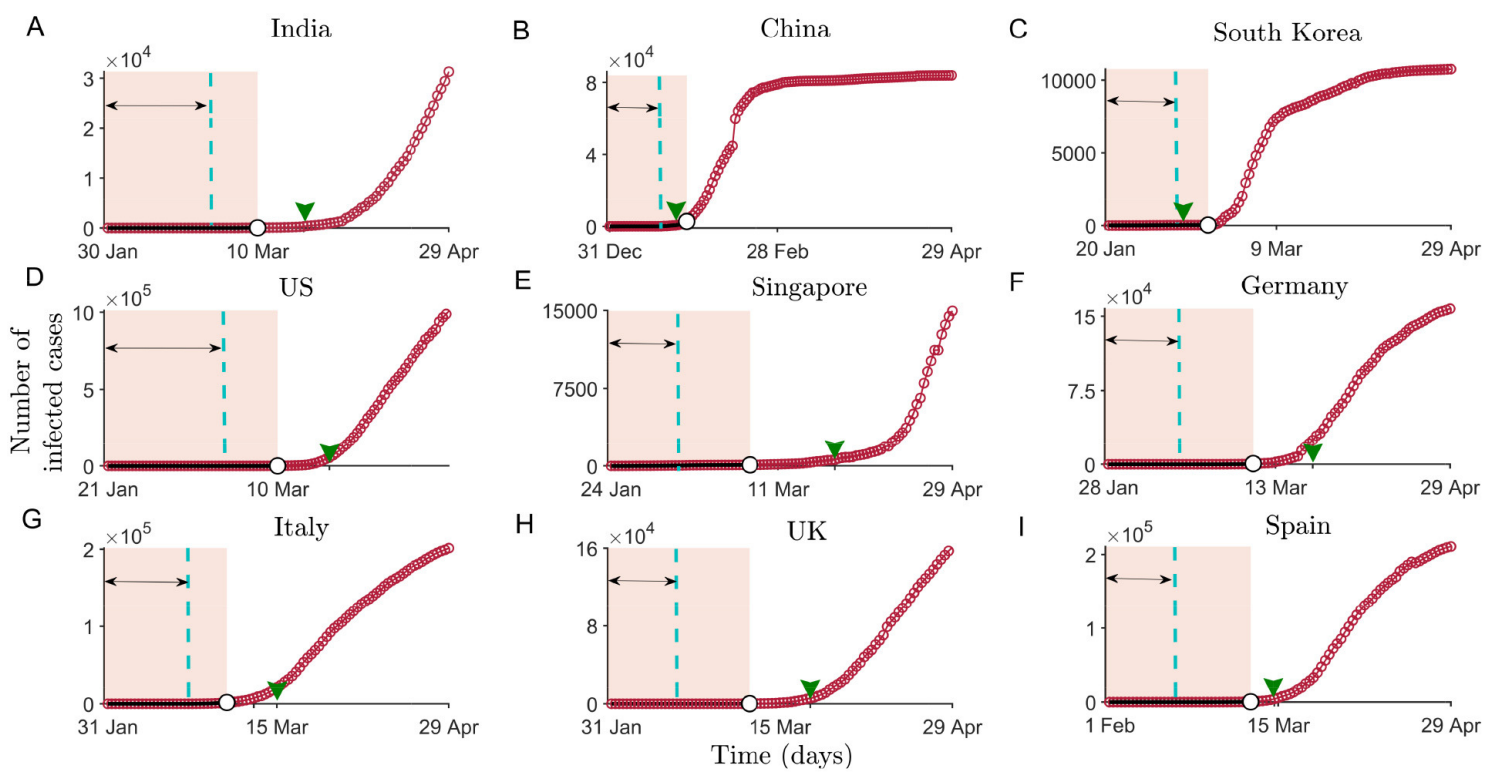

FIGURE 1 | Time series constructed as cumulative number of infected cases in nine different countries across the world from the onset of the epidemic in the respective countries up to April 29. (A-I): Shaded regions depict the pre-transition phase for different countries from the onset and mark the data used to compute the indicators of critical slowing down. The double-sided arrow marks the size of the moving window (up to the vertical dashed line). In the subfigures, the arrowhead on the $x$-axis marks the beginning of the officially recorded social-distancing and/or lockdown dates (see Table S1 in Supplementary Material).

implementation of social distancing measures. China was the first country to take the containment measures, nearly 24 days after the beginning of the epidemic, while Italy took around 40 days, and other countries followed even later. As a consequence, the COVID-19 incidence curve in China flattened after nearly 2025 days of implementing the intervention measures. Like China, South Korea adopted different combinations of controlling measures around mid-February (in the time window of 2025 days since the epidemic began there). This measure was accompanied by a drop in the number of cases, and the curve followed the pattern observed for China (Figure 1C). The rising indicators of CSD also suggest that the time gap in implementing the protocols, such as the closure of public gatherings, controlled public movement, and lockdown, can significantly influence the incidence curve and result in the extended time required to flatten it. However, the interventions around 2-3 weeks prior to the change in the correlation pattern as well as variance in each of these countries can slowly hamper the daily increase in the number of cases.

The scenario is quite different in the case of India. The EWSs weakly signal the behavior of CSD within the initial 40 days of the disease emergence (Figures $\mathbf{2 A}, \mathbf{J}$ ). Due to a rise in the number of daily cases, we also analyze the EWSs in the incidence curve for India considering the cumulative number of infected cases of up to the beginning of the nationwide lockdown (March 25, Figure 3A) since the reporting of the first case. Here, we observe increasing trend in each of the generic indicators capable of capturing the phenomenon of CSD. The variance, autocorrelation, skewness, and kurtosis captures the strong signals of CSD (Figures 3B-E).

\subsection{Onset of Social Distancing Practices and the Affected Population Density}

Another important aspect is to consider the reported proportion of a population affected at the time of the implementation of intervention measures. So far, Germany, which accounted for one of the largest outbreaks in Europe around mid-March, had visible signals of the forthcoming transition (Figures $\mathbf{2 F}, \mathbf{O}$ ). It is noted that each of the countries, namely India, Germany, and Italy, adopted concerned public health measures around the time when the EWSs were visible in their respective datasets (see the arrowheads on the $x$-axis in Figure 1). However, the fraction of the population affected by that time in Germany and Italy was much higher $\left(\sim 2.9 \times 10^{-4}\right.$ and $1.2 \times 10^{-4}$, respectively $)$ compared to India $-2.36 \times 10^{-7}$. Thus, the incidence curve projected a significant rise in these two countries, whereas the rise in the number of cases in India is relatively slow and is expected to follow a similar response owing to the effectiveness of these interventions. Overall, our analyzes suggest that delayed interventions (depending upon the signals of CSD) along with the fraction of the affected population can influence the countrywide variation in the daily number of rising cases.

\subsection{Sensitivity Analysis of the Generic Indicators}

The choices made to remove/filter out non-stationarities in the time series datasets using Gaussian detrending can also influence the trends observed. Thus, it is necessary to test the robustness of the estimated trends toward the choice of rolling window size and the filtering bandwidth. Here, we employ sensitivity analysis for the variance (see Figure 4) and $\mathrm{ACF}(1)$ 


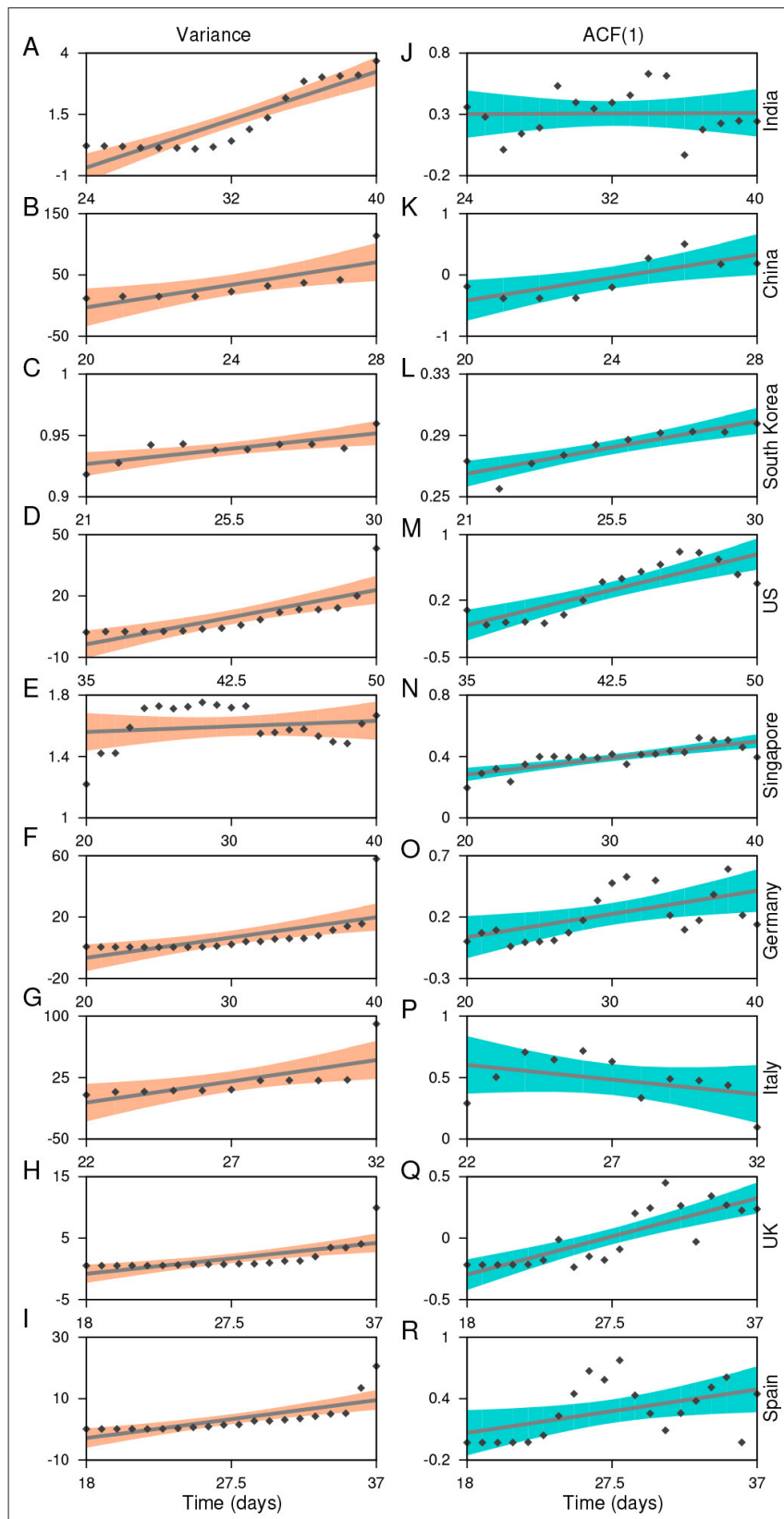

FIGURE 2 | Statistical estimates used to analyze the signals of a forthcoming transition in the COVID-19 incidence curve. (A-I): Figures on the left panel depicts the variance each for India, China, South Korea, USA, Singapore, Germany, Italy, UK, and Spain. (J-R): The right panel shows the lag-1 autocorrelation of the time series data analyzed in the corresponding countries. Scattered points are the estimated values of the respective slowing down indicators. Solid lines reflect the increasing/decreasing trend in the indicators and are obtained by fitting linear regression models. The shaded regions are the confidence bounds for the fitted models.

(see Figure 5) using the CSD dataset. Sensitivity analysis ease outs to disentangle accurate signals of an impending transition from the false ones for a wide range of window sizes and bandwidths. We use Kendall- $\tau$ estimates of these indicators for all the combinations of these two parameters (for details, see Materials and Methods). Furthermore, we test the sensitivity of

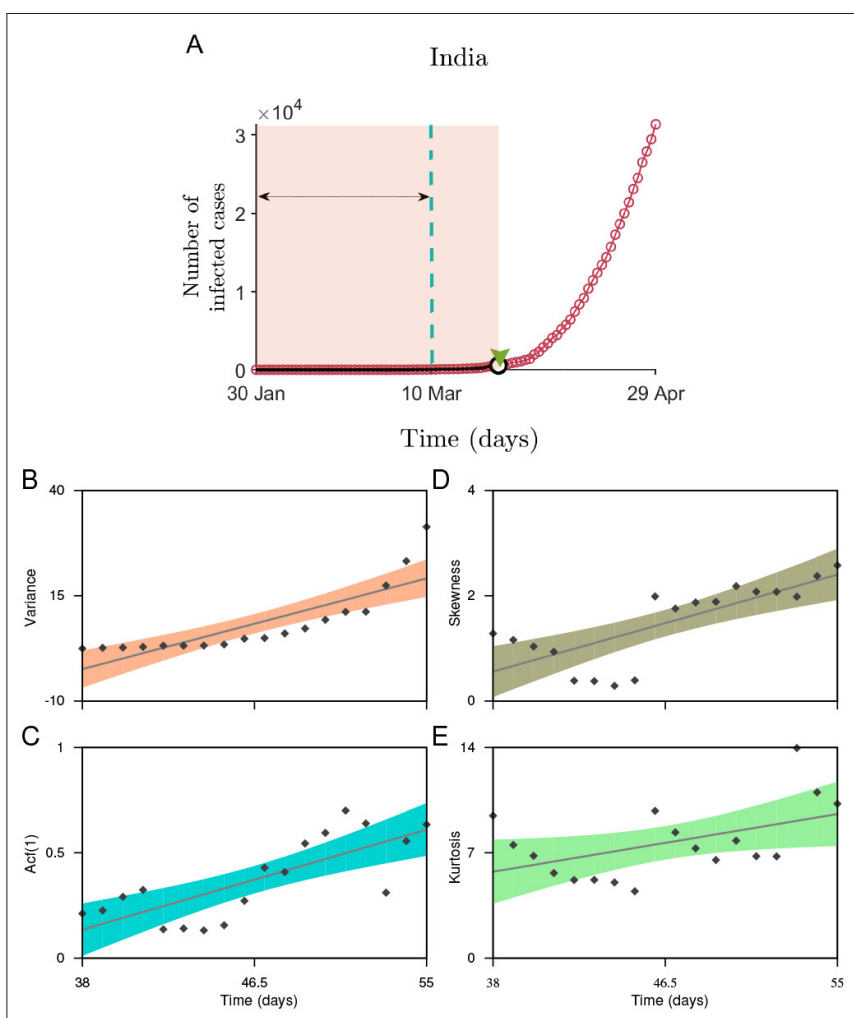

FIGURE 3 | Statistical analysis to measure the indicators of CSD for the dataset of India up to the date of the nationwide lockdown. (A) The incidence curve depicting the fraction of people infected from January 30 up to April 29. The shaded region is the data used to calculate the changes in the generic indicators. The arrow marks the size of the rolling window used to calculate the statistical signals. The estimated values of the slowing down indicators (B) variance, (C) autocorrelation function at lag-1, (D) skewness, and (E) kurtosis. Solid lines are the fitted linear regression models to analyze the trend in the indicators along with the confidence bounds (shaded regions).

these parameters on the $P$ values of the estimated indicators (for details see Section 3: Sensitivity Analysis and Figures S2,S3 in Supplementary Material).

We find that the observed trends in the variance are robust to the choice of parameters and does not vary between the datasets of most countries. High bandwidths reveal the opposite outcome of the variance for the datasets of South Korea (Figures 4C,L) and Singapore (Figures 4E,F). Since we use the bandwidth, which gives the best fit and does not over-fit or under-fit the data therefore, the choice of window size can influence the observed trends. In our work, we find a large size of rolling window can alter the EWSs analysis and misleading estimates for the autocorrelation function (Figures $5 \mathbf{A}-\mathbf{N}$ ). False signals of an alarming situation can deviate from understanding the gravity of any situation and intensity of surveillance needed. Thus, the choice of these parameters is crucial in anticipating the signals of a forthcoming transition and implementing convincing public health measures.

\subsection{Surrogate Analysis}

The lower number of data points available for the analysis can lead to feeble trends and influence the probability of 


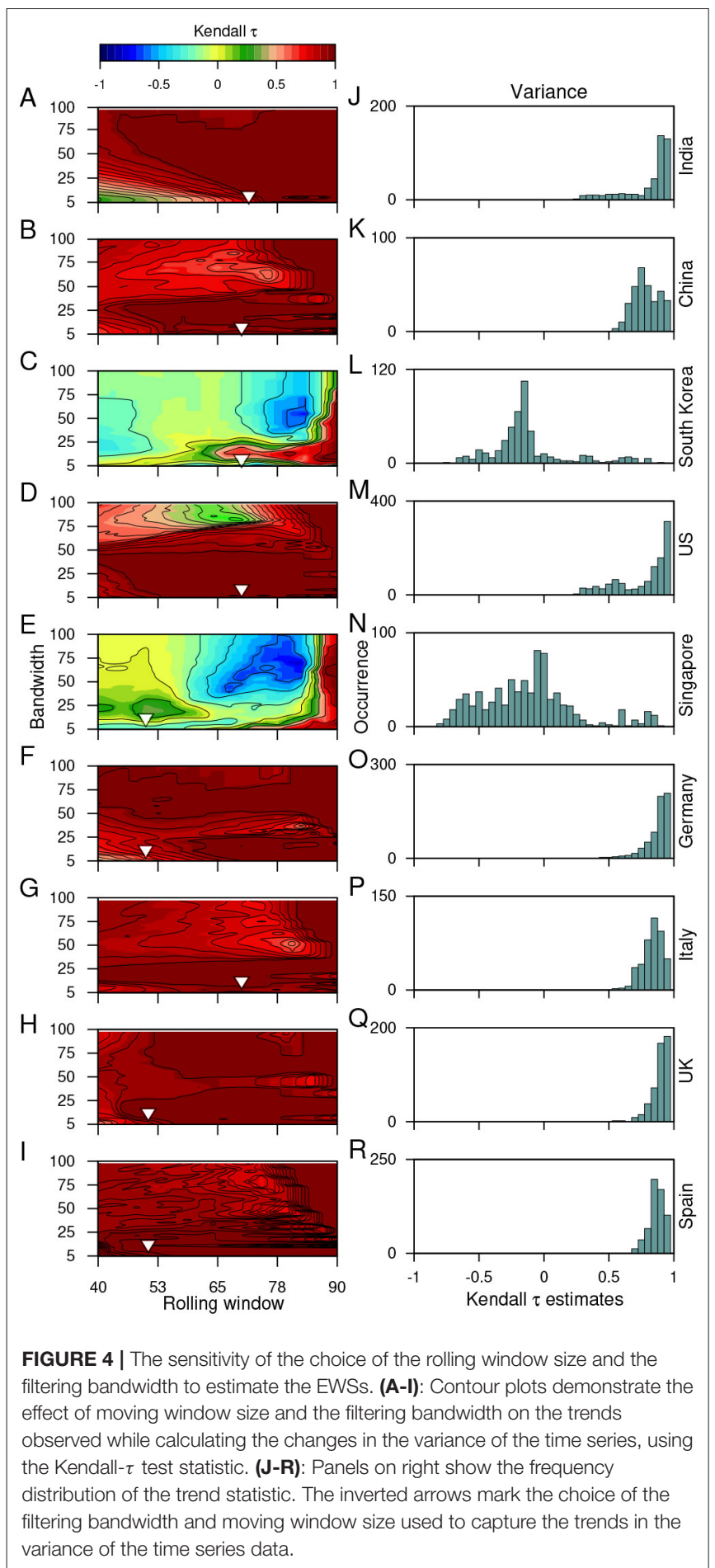

occurrence of the increased signals of CSD by chance. Further, due to undocumented patients, there is always a chance of stochasticity in the number of reported cases. Thus, we studied the likelihood of coincidence in the occurrence of trends in the variance and the $\mathrm{ACF}(1)$ observed in our original datasets by investigating the indicators in the surrogate time series (see Materials and Methods). The surrogate time series is

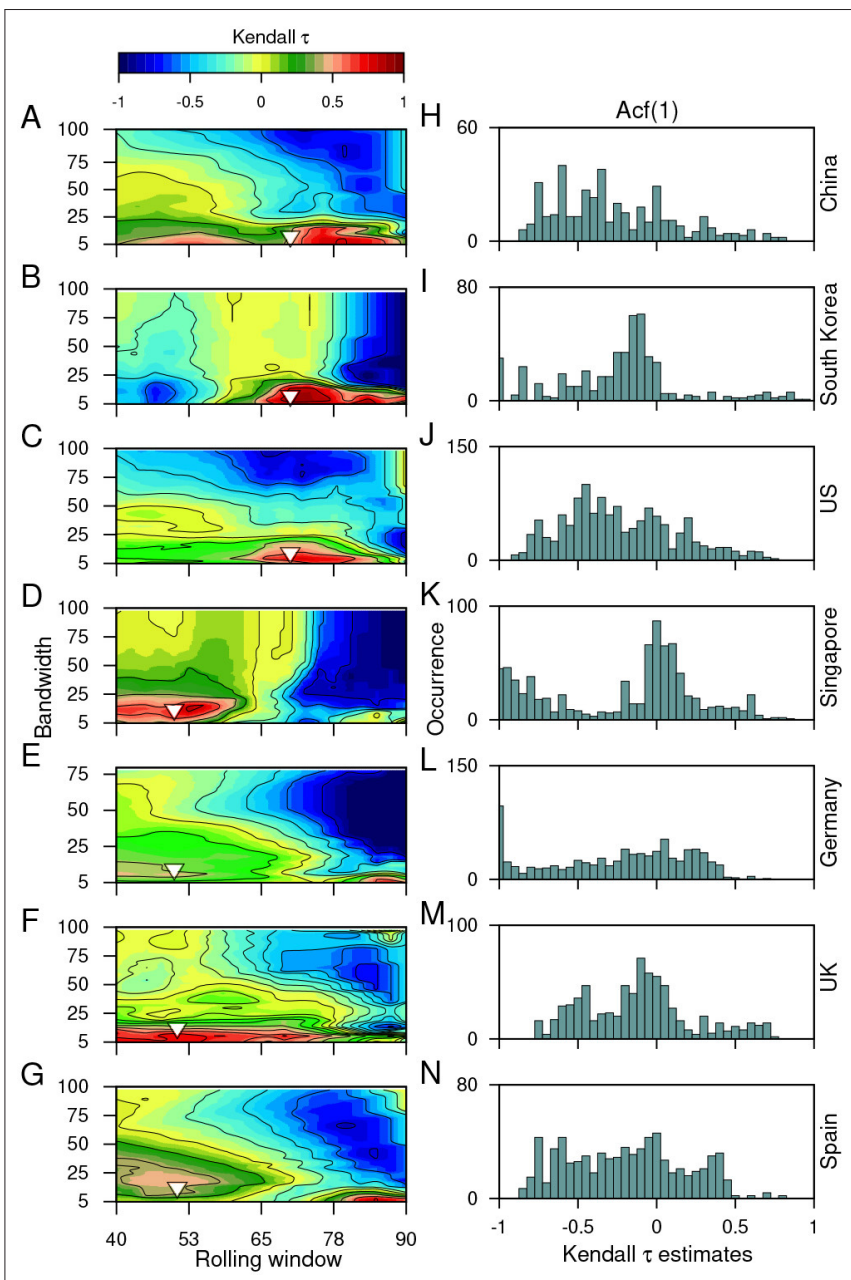

FIGURE 5 | The sensitivity of the choice of the rolling window size and the filtering bandwidth to estimate the EWSs. (A-G): Contour plots demonstrate the effect of moving window size and the filtering bandwidth on the trends observed while calculating the short term correlation pattern using $\operatorname{ACF}(1)$ of the time series data, using the Kendall- $\tau$ test statistic. (H-N): Panels on right show the frequency distribution of the trend statistic. The inverted triangles mark the choice of the filtering bandwidth and moving window size used to capture the trends in the $\mathrm{ACF}(1)$ of the time series data.

generated to follow similar distribution (mean and variance) of the data time series before the episode of a sudden rise in the number, denoted by shaded regions in Figure 1 (see Materials and Methods). Figure 6 depicts the distribution of the test statistic of the surrogate time series. Solid lines show the trend estimate obtained for the original time series. We calculate the probability of randomness of our observed estimates as the fraction of 1,000 surrogate time series having trend statistic of same or higher values than the original trend, i.e., $P\left(\tau^{*} \leq \tau\right)$. The probability of, by chance, obtaining similar trend statistic varies from country to country, depicting significant estimates for changes in the variance, except for South Korea (Figures 6B,I) and Singapore (Figures 6D,K). In the case of the US, however, the probability of randomness in our observed estimates is lower (Figures $\mathbf{6 C}, \mathbf{J}$ ), and rapid spreading in the 


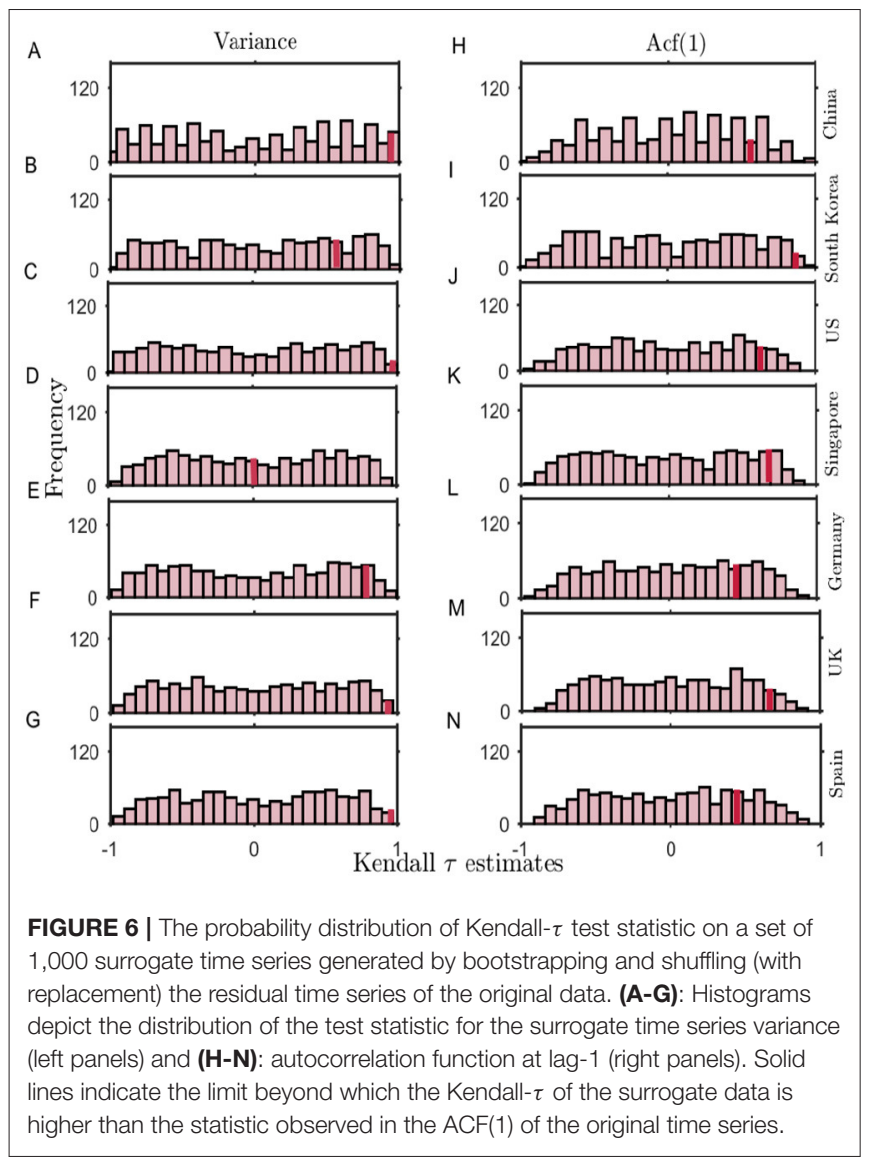

TABLE 1 | Probability of, by chance, obtaining the observed trend statistic of the original data for the set of 1,000 surrogates having similar distribution (mean and variance) as the original datasets.

\begin{tabular}{lcc}
\hline Country & $\begin{array}{c}\text { Kendall- } \boldsymbol{\tau} \\
\text { (variance) }\end{array}$ & $\begin{array}{c}\text { Kendall- } \boldsymbol{\tau} \\
\text { [ACF(1)] }\end{array}$ \\
\hline China & 0.04 & 0.09 \\
South Korea & 0.21 & 0.01 \\
US & 0.001 & 0.1 \\
Singapore & 0.48 & 0.09 \\
Germany & 0.08 & 0.20 \\
UK & 0.001 & 0.05 \\
Spain & 0.001 & 0.21
\end{tabular}

The likelihood of randomness in the estimated variance and $A C F(1)$ is mentioned for the datasets of each country studied in the work.

epidemic makes it keystone to consider applicability of EWSs to warn-off such events. The probability estimates $P$ obtained by bootstrapping the datasets for each of the countries are given in Table 1.

Overall, we find a low probability of randomness in both the $\operatorname{ACF}(1)$ and the variance estimates for most of the cases. However, the observations are more significant for the variance. This analysis suggests the robustness of the variance as an EWS in predicting the signals of CSD.

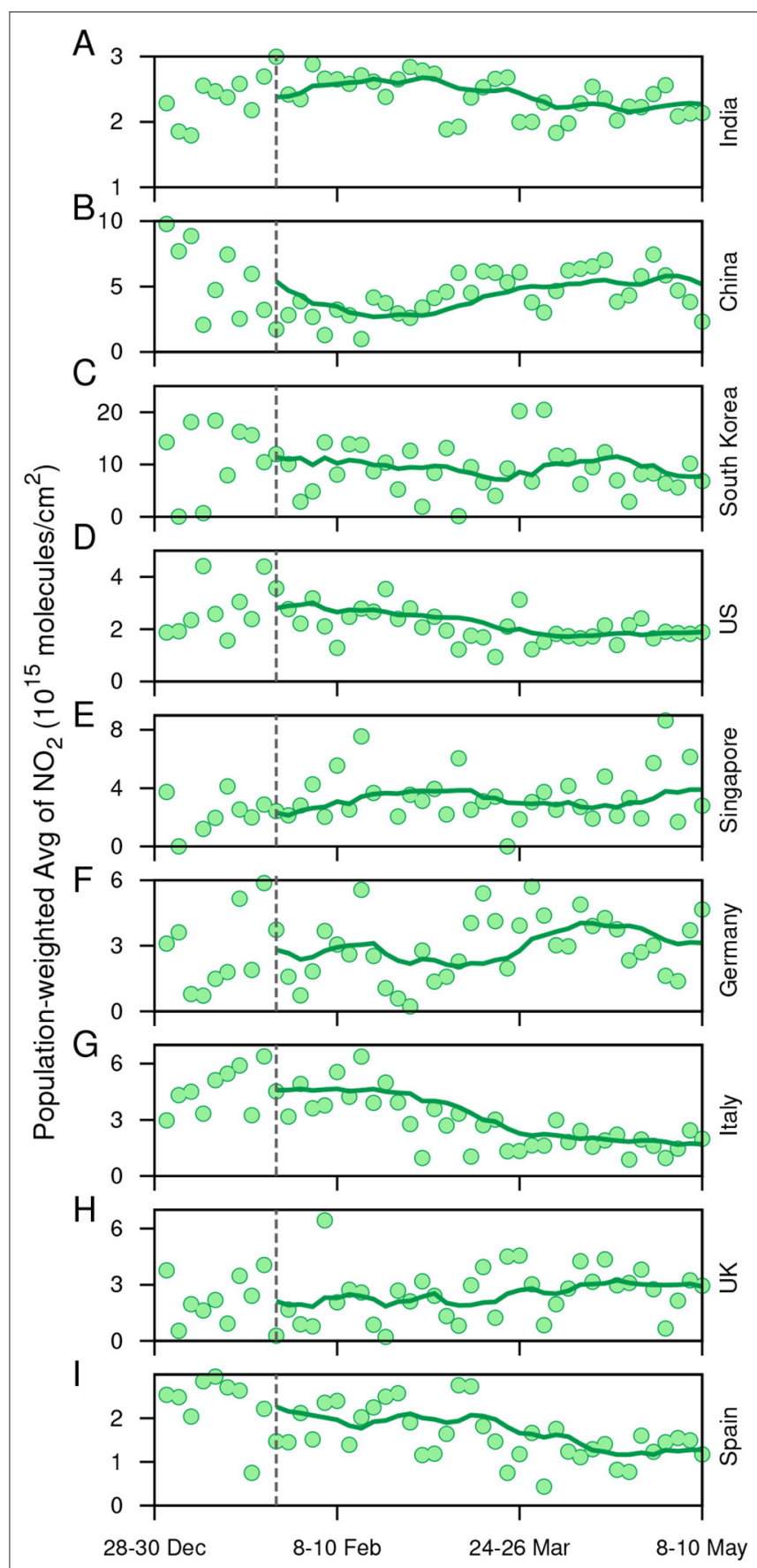

FIGURE 7 | (A-I): Time series of triads of the population-weighted total-column $\mathrm{NO}_{2}$ (molecules $/ \mathrm{cm}^{2}$ ) density over the length of the study period for the nine countries considered in this work (depicted by the circular points). The solid curve in each subfigure represents a 10-triad moving window average of the time series.

\subsection{Impact of COVID-19 Spread on the Atmospheric Total-Column $\mathrm{NO}_{2}$ Density}

The rigor of social distancing/intervention strategies can be measured by atmospheric data, as the lockdown periods have 
witnessed better air quality across the globe (39). We note that anthropogenic $\mathrm{NO}_{2}$ is emitted predominantly at the surface from transportation activities, industries, and power plants. $\mathrm{NO}_{2}$ emitted has a short lifetime and can be transported up to a few hundred meters during the day. Therefore, $\mathrm{NO}_{2}$ is expected to be a profound indicator of the efficiency of lockdown measures enforced by the countries. Thus, we first obtain time series of triads of the population-weighted total-column $\mathrm{NO}_{2}$ (molecules $/ \mathrm{cm}^{2}$ ) density over the length of the study period for the nine countries considered in this work (the circular points in Figure 7) (for the $\mathrm{NO}_{2}$ data source see Materials and Methods). The solid curve in each subfigure of Figure 7 represents a 10-triad moving window average. In the majority of the countries, the timing of $\mathrm{NO}_{2}$ decline concurs with the spread of the virus and the onset of pragmatic lockdown in a country may be hypothesized by the reversal (or break) in the trend of $\mathrm{NO}_{2}$. In China (Figure 7B), the decreasing trend in $\mathrm{NO}_{2}$ is evident from January to February; after that, it starts increasing which is coincident with the dynamics of the spread of COVID-19 disease. In India (Figure 7A), South Korea (Figure 7C), US (Figure 7D), Italy (Figure 7G), and Spain (Figure 7F), the decreasing trend in $\mathrm{NO}_{2}$ coincides with time of the rapid spread in the virus (Figure 1). We estimate that after the date of official enforcement of lockdown, the time-averaged $\mathrm{NO}_{2}$ decreased by $26.6 \%$ in China and $55.6 \%$ in Italy compared to the pre-lockdown period. Spain, USA, and India have also seen a significant decrease after the lockdown was enforced in these countries by $33,22.9$, and $11.8 \%$, respectively. It increased in the UK and Germany by 18 and 32\%, respectively, however, even after the initiation of lockdown, which indicates an inefficient closure of anthropogenic activities (like road and rail transport, industries, and power plants). The spatial distribution of totalcolumn $\mathrm{NO}_{2}$ for all the triads from Dec 28, 2019, to May, 10, 2020, can be visualized Movie 1 in Supplementary Material. It should be noted that we did not control for meteorological variations, which may have a significant impact on total-column $\mathrm{NO}_{2}$ over the period of our study (40). Overall, amidst the fears of the novel coronavirus, the countries where the lockdown intervened are expecting a rejuvenated environment. However, at the same time, possibilities of decreasing air pollutants when the world is not facing such harsh conditions is also important to understand.

\subsection{A Minimal Stochastic Model}

We propose a minimal kinetic model for the short-term prediction of the spreading of COVID-19 disease. Suppose that the only processes are infection and recovery. The processes can be described as

$$
I+H \stackrel{k_{i}}{\rightarrow} I+I ; \text { and } I \stackrel{k_{r}}{\rightarrow} H
$$

where $I$ and $H$ are infected and healthy people, respectively, and $k_{i}$ and $k_{r}$ are rate constants for infection and recovery. The first equation shows that if $I$ is the infected people, then $H$ becomes $I$ at a rate $k_{i}$; and the second equation indicates that $I$ recovers at a rate $k_{r}$. A minimal kinetic model can be formulated as ordinary differential equations for the population of $I$ :

$$
\frac{d I}{d t}=k_{i} I\left(1-\frac{I}{K}\right)-k_{r} I
$$

where $K$ is the size of the population.

We develop a master equation for the infected population by considering the two elementary processes (Equation 1). The transition probability at which the number of infected population increases from $i$ to $(i+1)$ is $w(i+1 \mid i)=k_{i} i(1-i / K)$, and the rate at which the number of infected population reduces from $i$ to $(i-1)$ is $w(i-1 \mid i)=k_{r} i$. From these, the probability of finding $i$ infectives in the system at time $t, P(i, t)$ can be obtained from the following equation:

$$
\begin{aligned}
\frac{d P(i, t)}{d t}= & w(i \mid i-1) P(i-1, t)+w(i \mid i+1) P(i+1, t) \\
& -(w(i+1 \mid i)+w(i-1 \mid i)) P(i, t) .
\end{aligned}
$$

The above probabilistic model is solved by the kinetic Monte Carlo simulations by means of the Gillespie algorithm, which incorporates the intrinsic noise (41). The algorithm considers each of the events as individual realizations of the Markov process. The time and species numbers are updated stochastically by choosing the random processes.

To simulate the system (Equation 3), we first obtain the parameters from the cumulative time series data of confirmed cases for India, China, and South Korea. In the datasets, we fitted the below logistic function (which is a solution of Equation 2):

$$
f(t)=\frac{a}{1+b \exp (-c t)},
$$

where $a, b$, and $c$ are parameters. Once we obtain these parameters for an individual country, we map them to our model and find the system parameters $k_{i}, k_{r}$ and $K$, and $i_{0}$ is the initial infected population. We list those parameters below:

\begin{tabular}{|l|l|l|l|l|}
\hline Country & $\boldsymbol{k}_{\boldsymbol{i}}$ & $\boldsymbol{k}_{\boldsymbol{r}}$ & $\boldsymbol{K}$ & $\boldsymbol{i}_{\mathbf{0}}$ \\
\hline India & 0.608 & 0.486 & $11,722,830$ & 2 \\
\hline China & 1.235 & 0.988 & 419,880 & 27 \\
\hline S. Korea & 0.9075 & 0.726 & 54,200 & 4 \\
\hline
\end{tabular}

Then the above parameters are used to solve the Master equation (Equation 3), and we perform Monte-Carlo simulation to get stochastic trajectories up to April 15. We present the simulated stochastic trajectories in Figure 8. For each country, we have five trajectories. For China and South Korea, we find that our stochastic trajectories are consistent with the real time series of the number of infected people. However, for India, our result shows that on May 20, 2020, the number of infected people reached $\sim 109,262$ (an average of final values of the five simulated trajectories).

The problem of predicting the spreading of COVID-19 is a complex one and depends on many factors like social distancing, an early detection of the disease, the detection of major hubs of the disease, etc. Here, we have provided a minimal kinetic model that uses the trends of the available data and may work only for short term prediction. 

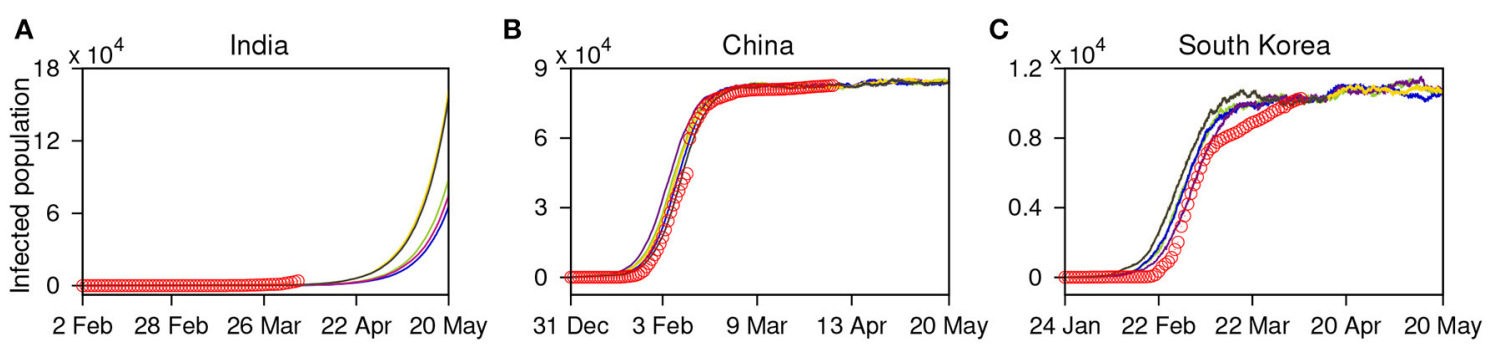

FIGURE 8 | Stochastic trajectories (marked with pink, yellow, gray, green, and blue curves) of the infected population generated using the Gillespie algorithm for: (A) India, (B) China, and (C) South Korea. The original datasets up to April 6 for the respective countries are depicted by circular (red) points for a comparison.

\section{DISCUSSION}

The COVID-19 pandemic revealed an exponential rise in the reported number of cases and has affected the public health, ranging from mild to severe conditions. Countries across the world are combating the spread of the coronavirus through various social distancing/intervention measures, such as the closure of schools and universities, banning of public events and large gatherings, isolation of symptomatic COVID-19 cases, implementation of mass quarantines, etc. For national as well as international control of public health, it is crucial to understand the significance of the onset timing of such measures (42).

The World Health Organization lately reported new cases being detected in several new countries across the globe (43-45). Our study can provide insight to tackle the ongoing pandemic and its associated incidence curve in the context of the timing and strength of the interventions. We use the data of the number of COVID-19 cases in nine different countries to investigate some statistical patterns in the incidence curves. The number of cases covers a small fraction of the population during the initiation of the epidemic, and the fraction remains nearly stagnant ranging nearly from 20 to 50 days from the arrival of the first case. Furthermore, the number of cases are increasing rapidly, and in a relatively shorter span, a significant fraction of the population can be affected. This trend is analogous to the idea that the incidence curve remains close to one stable state for a sufficient time and, crossing a time threshold, invokes a sudden shift/transition to another stable state, where a significant fraction of the population gets affected. In our work, we employ statistical indicators of critical slowing down to check if such transitions can be signaled beforehand and how the anticipation of such transitions can help mitigate such a crisis at a policy level.

We observe that the initial time window from the arrival of the first case in each country signaled an impending transition. An increase in the $\mathrm{ACF}(1)$ of the data as well as variance, before an actual rise in the number of cases, indicates the phenomenon of critical slowing down. Our work suggests that while non-pharmaceutical interventions are necessary to mitigate such an epidemic, the timing of initiation of concerned actions can strongly influence the outcome of the situation. Owing to the time lag in the detection of symptomatic cases, the statistical indicators suggest that a time period of 2-3 weeks before an impending transition is crucial to suppress the loss of public health. The controlled response of the epidemic incidence curve for China and South Korea can be associated with the time distance between implementation of interventions and the transition point. Both these countries initiated interventions before the visible signals of CSD in the incidence curve. Timely interventions were thus important factors to suppress the fluctuations in the number of cases and shape the curve. The analysis of EWSs analysis is crucial while defining the onset of the interventions and suppress the rise in daily cases. Importantly, another crucial aspect is the proportion of affected cases in each country, i.e., a measure of the fraction of the country's population, and not the absolute numbers, which is infected at the time of interventions, such as a strict lockdown. As probability of the propagation of disease can be thought of as mostly similar or equal amongst individuals across the globe, it depends upon the fraction of infected cases in each country during the beginning of interventions. For instance, the EWS analysis anticipated the upcoming rise in the incidence curves for both India as well as Italy, and, interestingly, both the countries imposed individual nationwide lockdown near the situation close to the transition (see Table S1 in Supplementary Material). However, the control in India depicts better results in altering the incidence curve than that in Italy. The alterations in the incidence curve is most likely to be a consequence of a difference in the proportion of cases affected by the epidemic at the beginning of mitigation strategies. India, resembling China in terms of the total population density, accounted for $\sim 2.36 \times 10^{-7}$ cases of the total population, while Italy, with a relatively smaller population density, crossed $1.22 \times 10^{-4}$ cases of their total population. Thus, even with imposition of the public health measures near the signals of CSD, the outcome for both the countries can vary dramatically. The variation is the consequence of the proportion of affected cases when visible signals of EWSs are observed and at the time of interventions. This suggests that the proportion of affected population during visible signals of CSD is key to shaping the disease incidence curve. The strength of the signals can alter the duration and scale of the interventions needed. Furthermore, the disruptive situation in the US is indicated by EWSs, as the EWSs indicators show significant trends for the US in addition to a large fraction of population being affected at that time. A sharp rise in the number of cases for the country is a consequence of both the delay in effective social distancing interventions as well as a significant proportion of affected cases. Overall, our work 
suggests that, in almost all the countries, an imminent sharp rise in the incidence curve can be seen using statistical measures prior to the actual transition.

Another issue the infectious coronavirus raises is the quality of air pollution in countries where social distancing/lockdown is enforced. $\mathrm{NO}_{2}$, which is majorly emitted from anthropogenic activities like land transportation, industries, and energy sectors, was estimated to decrease in consequence to lockdown measures implemented by the government of respective countries. Population-weighted average column $\mathrm{NO}_{2}$ was found to decrease with amplification in a number of cases across most of the countries. Apart from this, $\mathrm{NO}_{2}$ column quantities may be used as a proxy to estimate the effectiveness of a lockdown on air quality. We find that $\mathrm{NO}_{2}$ column quantities started following a decreasing trend during the last week of February in Italy and the US, which indicates a partial unofficial closure of anthropogenic activities, taking into consideration that the official COVID-19induced lockdown was enforced on March 09 and around March 25 in Italy and the US, respectively. In the UK, however, an increasing trend in the $\mathrm{NO}_{2}$ column up until May 10 indicates no such public awareness to restrict anthropogenic activities (the government declared the lockdown from March 23). We acknowledge that the reduction in $\mathrm{NO}_{2}$ is also associated with the compliance of the population of the individual nation to abide by the lockdown measures.

Furthermore, we suggest that the interventions employed by India may not come at a time when the curve is very far from reaching the transition; however, the smaller number of affected cases may be the determining factor in limiting the disease spread in India. Implementation of a nationwide lockdown in India may have better prepared the country for taking measures to control the epidemic spread and bend of the curve. However, our analysis also suggests that the period beyond the signals of CSD also needs efficient monitoring. The results of our minimal stochastic model predicted that, on May 20, the number of infected people could go up to $\sim 109,262$. Thus, an extended period of such measures is needed and likely to be effective (6).

We envision that it is fundamental to identify the situation of such a crisis across the world and make use of the lead time. The EWSs can keep track of the changes in the trend statistics in the number of reported cases and warn when a threshold is reached. The statistical tools used can be beneficial to identify whether the features of shift in a system are suppressed by the intervention strategies being adopted. In particular, while different combinations of strategies are adopted to overcome such a crisis, the information of an upcoming transition and its threshold is important to formulate the degree of such interventions. However, special care should be taken in the choice of rolling window size and the filtering bandwidth while estimating the signals of slowing down. Inappropriate choices may give weak and/or diminished signals of an imminent transition, which may deviate from understanding the urgency of the situation. Another aspect to consider is that the varying extent of testing for COVID-19 across the countries may have affected the total number of reported cases; thus, our results here hold specifically for the number of reported cases.

\section{MATERIALS AND METHODS}

\subsection{The COVID-19 Data Source}

We have used the COVID-19 dataset provided by the European Centre for Disease Prevention and Control (ECDC): An agency of the European Union (available from https://www.ecdc.europa. eu/en/publications-data). Initially, we extract the data of the daily number of reported cases up to March 25, 2020, and in general mark the first date of the reported cases as the day of the beginning of the epidemic in the respective countries. Regardless of the affected person recovers or dies, the virus contraction occurs once; we thus consider cumulative data of the daily number of the confirmed cases for nine different countries for our study.

\subsection{Data Selection}

We use the available time series to test the predictability of an upcoming transition for each country. The generic indicators are examined using the time series segments before the transition in the number of cases of the epidemic (see Section 4: Detection of the Transition Phase and Figure S5 in Supplementary Material) in each country (shaded regions in Figure 1).

\subsection{Detrending}

Often, non-stationarities in the data lead to false indications of impending transitions. To overcome this, we obtain the residual time series by subtracting a Gaussian kernel smoothing function from the empirical time series (23). Furthermore, we estimate the variance and autocorrelation at first lag for the residual time series choosing a rolling window size from the sensitivity analyzes of the time series data for each country. We choose the filtering bandwidth and avoided any under-fit or over-fit (for details see Table S2 in Supplementary Material).

\subsection{Autocorrelation at First Lag and Cariance}

The fluctuations in the time series reveal different novel phenomena, such as sudden transition, flickering, stochastic switching, etc. It is established that followed by a perturbation, the rate of return of the system slows down near an impending transition or a tipping point. This phenomenon of slow return rate or recovery from a perturbation in the vicinity of a sudden transition is known as critical slowing down (CSD). We capture the signals of CSD by estimating changes in the short-term autocorrelation (at lag-1) and variance of the time series. CSD increases the short-term memory of the time series, which is observed through the correlation structure of the time series before a transition. We compute autocorrelation at lag-1 by fitting an autoregressive model of order 1 (of the form $z_{t+1}=$ $\left.\alpha_{1} z_{t}+\epsilon_{t}\right)$ using an ordinary least-squares fitting method. The time series analysis has been performed using the "Early Warning Signals Toolbox" (http://www.early-warning-signals.org/).

\subsection{Sensitivity Analysis}

The predictability of each of the indicator depends upon the datasets investigated as well as the choices made for processing 
the data. Thus, it is essential to check the efficacy of our results to such choices. In particular, we analyze the sensitivity of our observations to the choice of rolling window size and degree of smoothing (filtering bandwidth) used during the calculation of indicators and detrending/filtering the datasets, respectively. We estimate the CSD indicators using window sizes ranging from 40 to $90 \%$ of the time series length in an increment of 1 point and for bandwidths ranging from 5 to $100 \%$ with the increment of 1 point. We quantify the robustness of the outcomes toward the range of window sizes and bandwidth using the distribution of the Kendall- $\tau$ test statistic.

\subsection{Surrogates}

To test the significance of our statistical analysis, we estimate Kendall rank correlation- $\tau$ test statistics for both the generic indicators. We generate 1,000 surrogate time series of the same length as the analyzed real datasets to test the likelihood of obtaining the computed trends by chance. The surrogate records are obtained on bootstrapping the real datasets by shuffling the original residual time series and sampling the data with replacement. This method generates the surrogate time series with a similar distribution of the original time series (27). For each surrogate, we consider the Kendall- $\tau$ estimate as the test statistic to measure the robustness of the outcomes. Furthermore, we calculate the fraction of the surrogates having the same or higher test static value than the original data and measure the probability $P\left(\tau^{*} \leq \tau\right)$ to calculate that the observed test statistic is by chance. We also generate surrogate time series using phase randomization method (for details see Section 3: Surrogate Analysis and Figure S4 in Supplementary Material).

\subsection{Satellite Retrieved Total Column $\mathrm{NO}_{2}$}

Worldwide, the lockdown response to the onset and spread of COVID-19 caused a decrease in daily and economic activities, which in turn is expected to cause a reduction in ambient air pollution. This can also be used as an indicator to determine whether government policies of lockdowns/restricted human movements are successful or not. To further examine this, we use the Ozone Monitoring Instrument (OMI) retrieved total column $\mathrm{NO}_{2}$ (available from https://aura.gsfc.nasa.gov/omi.html) as a proxy to infer the change in anthropogenic air pollution for

\section{REFERENCES}

1. World Health Organization. Coronavirus Disease 2019 (covid-19): Situation Report, 59. Technical report, World Health Organization (2020).

2. Eurosurveillance Editorial Team. Note from the editors: World health organization declares novel coronavirus (2019-ncov) sixth public health emergency of international concern. Eurosurveillance. (2020) 25:200131e. doi: 10.2807/1560-7917.ES.2020.25.5.200131e

3. Covid-19 Coronavirus Pandemic. (2020). Available online at: https://www. worldometers.info/coronavirus/

4. Wu JT, Leung K, Bushman M, Kishore N, Niehus R, de Salazar PM, et al. Estimating clinical severity of covid-19 from the transmission dynamics in Wuhan, China. Nat Med. (2020) 26:506-10. doi: 10.1038/s41591-0200822-7 the time-period of our study. OMI flies onboard the EOS Aura sun-synchronous polar-orbiting satellite. It has a swath length of $2,600 \mathrm{~km}$ and a level-2 and spatial resolution of $13 \times 24 \mathrm{~km}^{2}$ (46). The $\mathrm{OMI} \mathrm{NO}$ column was satisfactorily validated against surface spectrometer measurements in recent studies $(47,48)$. To roughly obtain a global coverage, we consider 3-days time slices (triads) within which the overlapping swath overpasses were averaged. Thereafter, we perform a population-weighted average of the grids that lie within the political boundaries of the countries considered in this study. Gridded population data was obtained for 2015 from SEDAC (https://sedac.ciesin.columbia. edu/data/collection/gpw-v4).

\section{DATA AVAILABILITY STATEMENT}

All datasets presented in this study are included in the article/Supplementary Material.

\section{AUTHOR CONTRIBUTIONS}

SC, SKS, MJ, and PD designed the research. TK, SS, SC, SKS, and PD performed the research. TK, SS, SC, SKS, MJ, and PD analyzed the data. TK, SC, SKS, MJ, and PD wrote the paper. All authors contributed to the article and approved the submitted version.

\section{ACKNOWLEDGMENTS}

SS acknowledges the financial support from the Department of Science \& Technology (DST), Government of India, under the scheme DST-Inspire (Grant No: IF160459). PD acknowledges financial support from the Science \& Engineering Research Board (SERB), Government of India (Grant No: CRG/2019/002402). This manuscript has been released as a pre-print at medRxiv (49).

\section{SUPPLEMENTARY MATERIAL}

The Supplementary Material for this article can be found online at: https://www.frontiersin.org/articles/10.3389/fpubh. 2020.569669/full\#supplementary-material 
10. Bootsma MCJ, Ferguson NM. The effect of public health measures on the 1918 influenza pandemic in us cities. Proc Natl Acad Sci USA. (2007) 104:7588-93. doi: $10.1073 /$ pnas.0611071104

11. Ferguson N, Laydon D, Gilani GN, Imai N, Ainslie K, Baguelin M, et al. Report 9: Impact of Non-pharmaceutical Interventions (NPIS) to Reduce Covid19 Mortality and Healthcare Demand. Technical report, Imperial College London (2020).

12. Anderson RM, Heesterbeek H, Klinkenberg D, Hollingsworth TD. How will country-based mitigation measures influence the course of the covid-19 epidemic? Lancet. (2020) 395:931-4. doi: 10.1016/S0140-6736(20) 30567-5

13. Prem K, Liu Y, Russell TW, Kucharski AJ, Eggo RM, Davies N, et al. The effect of control strategies to reduce social mixing on outcomes of the covid19 epidemic in Wuhan, China: a modelling study. Lancet Public Health. (2020) 5:e261-70. doi: 10.1016/S2468-2667(20)30073-6

14. O'Regan SM, Drake JM. Theory of early warning signals of disease emergence and leading indicators of elimination. Theor Ecol. (2013) 6:333-57. doi: 10.1007/s12080-013-0185-5

15. Brett TS, Drake JM, Rohani P. Anticipating the emergence of infectious diseases. J R Soc Interface. (2017) 14:20170115. doi: 10.1098/rsif.2017.0115

16. O'Dea EB, Drake JM. Disentangling reporting and disease transmission. Theor Ecol. (2019) 12:89-98. doi: 10.1007/s12080-018-0390-3

17. Drake JM, Brett TS, Chen S, Epureanu BI, Ferrari MJ, Marty É, et al. The statistics of epidemic transitions. PLoS Comput Biol. (2019) 15:e1006917. doi: 10.1371/journal.pcbi.1006917

18. Harris MJ, Hay SI, Drake JM. Early warning signals of malaria resurgence in Kericho, Kenya. Biol Lett. (2020) 16:20190713. doi: 10.1098/rsbl.2019.0713

19. Kefi S, Dakos V, Scheffer M, Van Nes EH, Rietkerk M. Early warning signals also precede non-catastrophic transitions. Oikos. (2013) 122:641-8. doi: 10.1111/j.1600-0706.2012.20838.x

20. Liu Y, Gayle AA, Wilder-Smith A, Rocklv J. The reproductive number of COVID-19 is higher compared to SARS coronavirus. J Travel Med. (2020) 27:taaa021. doi: 10.1093/jtm/taaa021

21. Dibble CJ, O’Dea EB, Park AW, Drake JM. Waiting time to infectious disease emergence. J R Soc Interface. (2016) 13:20160540. doi: 10.1098/rsif.2016.0540

22. Scheffer M, Carpenter SR, Lenton TM, Bascompte J, Brock WA, Dakos V, et al. Anticipating critical transitions. Science. (2012) 338:344-8. doi: 10.1126/science.1225244

23. Dakos V, Carpenter SR, Brock WA, Ellison AM, Guttal V, Ives AR, et al. Methods for detecting early warnings of critical transitions in time series illustrated using simulated ecological data. PLOS ONE. (2012) 7:e41010. doi: 10.1371/journal.pone.0041010

24. Wang R, Dearing JA, Langdon PG, Zhang E, Yang X, Dakos V, et al. Flickering gives early warning signals of a critical transition to a eutrophic lake state. Nature. (2012) 492:419. doi: 10.1038/nature11655

25. Scheffer M, Bascompte J, Brock WA, Brovkin V, Carpenter SR, Dakos V, et al. Early-warning signals for critical transitions. Nature. (2009) 461:53-9. doi: 10.1038/nature08227

26. Sarkar S, Sinha SK, Levine H, Jolly MK, Dutta PS. Anticipating critical transitions in epithelial-hybrid-mesenchymal cell-fate determination. Proc Natl Acad Sci USA. (2019) 116:26343-52. doi: 10.1073/pnas.1913 773116

27. Dakos V, Scheffer M, van Nes EH, Brovkin V, Petoukhov V, Held H. Slowing down as an early warning signal for abrupt climate change. Proc Natl Acad Sci USA. (2008) 105:14308-12. doi: 10.1073/pnas.0802430105

28. Scheffer M. Critical Transitions in Nature and Society. Princeton, NJ: Princeton University Press (2009).

29. May RM, Levin SA, Sugihara G. Complex systems: ecology for bankers. Nature. (2008) 451:893-5. doi: 10.1038/451893a

30. Scheffer M, Carpenter S, Foley JA, Folke C, Walker B. Catastrophic shifts in ecosystems. Nature. (2001) 413:591. doi: 10.1038/35098000

31. Scheffer M, Bolhuis JE, Borsboom D, Buchman TG, Gijzel SMW, Goulson D, et al. Quantifying resilience of humans and other animals. Proc Natl Acad Sci USA. (2018) 115:11883-90. doi: 10.1073/pnas.1810630115

32. Dakos V, Van Nes EH, d'Odorico P, Scheffer M. Robustness of variance and autocorrelation as indicators of critical slowing down. Ecology. (2012) 93:264-71. doi: 10.1890/11-0889.1
33. Dutta PS, Sharma Y, Abbott KC. Robustness of early warning signals for catastrophic and non-catastrophic transitions. Oikos. (2018) 127:1251-63. doi: 10.1111/oik.05172

34. Brett T, Ajelli M, Liu Q-H, Krauland MG, Grefenstette JJ, van Panhuis WG, et al. Detecting critical slowing down in high-dimensional epidemiological systems. PLoS Comput Biol. (2020) 16:e1007679. doi: 10.1371/journal.pcbi.1007679

35. Ribeiro SP, Dattilo W, Castro e Silva A, Reis AB, Goes-Neto A, Alcantara L, et al. Severe airport sanitarian control could slow down the spreading of covid-19 pandemics in brazil. medRxiv. (2020).

36. Matamalas JT, Gómez S, Arenas A. Abrupt phase transition of epidemic spreading in simplicial complexes. Phys Rev Res. (2020) 2:012049. doi: 10.1103/PhysRevResearch.2.012049

37. Linton NM, Kobayashi T, Yang Y, Hayashi K, Akhmetzhanov AR, Jung S, et al. Incubation period and other epidemiological characteristics of 2019 novel coronavirus infections with right truncation: a statistical analysis of publicly available case data. J Clin Med. (2020) 9:538. doi: 10.3390/jcm9020538

38. Li R, Pei S, Chen B, Song Y, Zhang T, Yang W, et al. Substantial undocumented infection facilitates the rapid dissemination of novel coronavirus (SARSCoV2). Science. (2020) 368:489-93. doi: 10.1126/science.abb3221

39. Venter ZS, Aunan K, Chowdhury S, Lelieveld J. Covid-19 lockdowns cause global air pollution declines. Proc Natl Acad Sci USA. (2020). 117:18984-90. doi: $10.1073 /$ pnas.2006853117

40. Jacob DJ, Winner DA. Effect of climate change on air quality. Atmos Environ. (2009) 43:51-63. doi: 10.1016/j.atmosenv.2008.09.051

41. Gillespie DT. Stochastic simulation of chemical kinetics. Annu Rev Phys Chem. (2007) 58:35-55. doi: 10.1146/annurev.physchem.58.032806.104637

42. Guest JL, del Rio C, Sanchez T. The 3 steps needed to end the covid-19 pandemic: Bold public health leadership, rapid innovations, and courageous political will. JMIR Public Health Surveill. (2020) 6:e19043. doi: 10.2196/19043

43. Coronavirus Disease 2019 (Covid-19) Situation Report 72. (2020). Available online at: https://www.who.int/docs/default-source/coronaviruse/situationreports/20200401-sitrep-72-covid-19.pdf?sfvrsn=3dd8971b_2

44. Coronavirus Disease 2019 (Covid-19) Situation Report 74. (2020). Available online at: https://www.who.int/docs/default-source/coronaviruse/situationreports/20200403-sitrep-74- covid-19-mp.pdf?sfvrsn=4e043d03 14

45. Coronavirus Disease 2019 (Covid-19) Situation Report 76. (2020). Available online at: https://www.who.int/docs/default-source/coronaviruse/situationreports/20200405- sitrep-76- covid-19.pdf?sfvrsn=6ecf0977_2

46. Krotkov NA, Lamsal LN, Celarier EA, Swartz WH, Marchenko SV, Bucsela EJ, et al. The version $3 \mathrm{OMI} \mathrm{NO}_{2}$ standard product. Atmos Meas Tech. (2017) 10:3133-49. doi: 10.5194/amt-10-313 3-2017

47. Wang Y, Beirle S, Lampel J, Koukouli M, De Smedt I, Theys N, et al. Validation of OMI. GOME-2A and GOME-2B tropospheric $\mathrm{NO}_{2}, \mathrm{SO}_{2}$ and HCHO products using MAX-DOAS observations from 2011 to 2014 in Wuxi, China: investigation of the effects of priori profiles and aerosols on the satellite products. Atmos Chem Phys. (2017) 17:5007-33. doi: 10.5194/acp-17-5007-2017

48. Lamsal LN, Krotkov NA, Celarier EA, Swartz WH, Pickering KE, Bucsela EJ, et al. Evaluation of OMI operational standard $\mathrm{NO}_{2}$ column retrievals using in situ and surface-based $\mathrm{NO}_{2}$ observations. Atmos Chem Phys. (2014) 14:11587. doi: 10.5194/acp-14-11587-2014

49. Kaur T, Sarkar S, Chowdhury S, Sinha SK, Jolly MK, Dutta PS. Anticipating the novel coronavirus disease (covid-19) pandemic. medRxiv. (2020).

Conflict of Interest: The authors declare that the research was conducted in the absence of any commercial or financial relationships that could be construed as a potential conflict of interest.

Copyright (c) 2020 Kaur, Sarkar, Chowdhury, Sinha, Jolly and Dutta. This is an open-access article distributed under the terms of the Creative Commons Attribution License (CC BY). The use, distribution or reproduction in other forums is permitted, provided the original author(s) and the copyright owner(s) are credited and that the original publication in this journal is cited, in accordance with accepted academic practice. No use, distribution or reproduction is permitted which does not comply with these terms. 